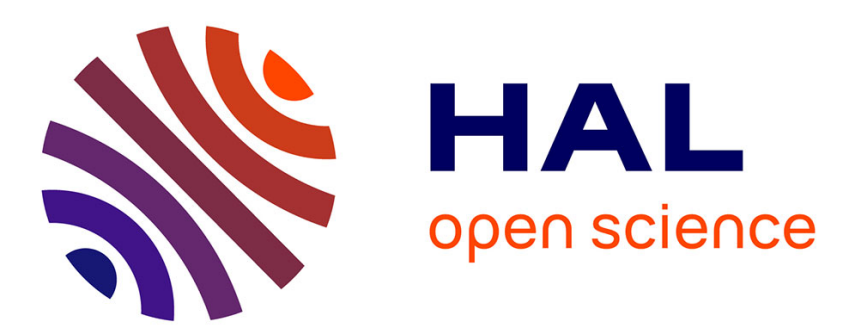

\title{
Light scattering by cooperative diffusion in semi-dilute polymer solutions
}

\author{
M. Adam, M. Delsanti, G. Jannink
}

\section{To cite this version:}

M. Adam, M. Delsanti, G. Jannink. Light scattering by cooperative diffusion in semi-dilute polymer solutions. Journal de Physique Lettres, 1976, 37 (3), pp.53-56. 10.1051/jphyslet:0197600370305300 . jpa-00231234

\section{HAL Id: jpa-00231234 https://hal.science/jpa-00231234}

Submitted on 1 Jan 1976

HAL is a multi-disciplinary open access archive for the deposit and dissemination of scientific research documents, whether they are published or not. The documents may come from teaching and research institutions in France or abroad, or from public or private research centers.
L'archive ouverte pluridisciplinaire HAL, est destinée au dépôt et à la diffusion de documents scientifiques de niveau recherche, publiés ou non, émanant des établissements d'enseignement et de recherche français ou étrangers, des laboratoires publics ou privés. 


\title{
LIGHT SCATTERING BY COOPERATIVE DIFFUSION IN SEMI-DILUTE POLYMER SOLUTIONS
}

\author{
M. ADAM, M. DELSANTI and G. JANNINK \\ Service de Physique du Solide et de Résonance Magnétique, \\ Centre d'Etudes Nucléaires de Saclay, \\ BP n $^{\circ} 2,91190$ Gif-sur-Yvette, France
}

(Reçu le 5 janvier 1976, accepté le 9 janvier 1976)

\begin{abstract}
Résumé. - La diffusion Rayleigh de la lumière par des solutions semi-diluées de polymères met en évidence une diffusion coopérative qui croît avec la concentration.

Abstract. - We report measurements of relaxation times of the autocorrelation function for the Rayleigh line of light scattered by semi-dilute polymer solutions.
\end{abstract}

Recent observations of polymer diffusive motions by light scattering have dealt with two extreme situations : the very dilute solution $[1,2]$, in which chains perform, as entities, diffusive motion, and the network [3] with fixed density of cross links. Here the deformation of the structure spreads out as a diffusive motion. From a systematic study of the effect of average molecular weight $M$ in between cross links, a remarkable property of this motion [3] was established in swollen networks : the diffusion coefficient $D_{\mathrm{S}}$, which is the ratio of elastic modulus to friction coefficient is inversely proportional to the power law of $M$ as found in the diffusion coefficient $D_{0}$ in very dilute solution. This suggests that the relevant quantity for the $M$ dependance of $D_{\mathrm{S}}$ is the average distance between cross links.

The semi dilute solution is an intermediate situation which has been considered by several authors [4, 5], as networks with a finite lifetime. Viscoelastic properties $[4,6]$ have been interpreted in this way. However, the situation was described as purely random, i.e. with total loss of hydrodynamic as well as excluded volume effects, so that the distance between neighbour cross links would scale in a trivial manner with concentration. De Gennes [7] has shown on the contrary that this distance reflects basic long chain properties and that the dynamical correlations in semi-dilute solutions are of fundamental interest. We shall try to present his arguments as follows :

1) There is a characteristic frequency $T_{R}^{-1}$, above which the solution can be considered as a network and below which it is seen as individual diffusive motions of the chains ; $T_{\mathrm{R}}$ depends upon molecular weight and concentration of the polymers. The range of values taken by $T_{\mathrm{R}}$ is well suited to an expe- riment on light scattering by diffusive network deformation.

2) As in the permanent network, the diffusion coefficient associated with deformation, $D_{\mathrm{c}}$, depends upon the average distance between adjacent cross links. However, this length does not depend upon molecular weight of the chains. It is only a function of concentration

$$
D_{\mathrm{c}}=k_{\mathrm{B}} T / 6 \pi \eta_{0} \xi
$$

where $k_{\mathrm{B}}$ is the Boltzman constant, $T$ the temperature, $\eta_{0}$ the viscosity of the solvent and $\xi$ the dynamical screening length, identical to the static characteristic correlation length [8]

$$
\xi \propto C \frac{v}{1-v d}
$$

where $v$ is the excluded volume exponent $\left(v=\frac{3}{5}\right.$ in three dimensions) and $d$ the dimension of space. De Gennes [7] established this result as a consequence of two non trivial identifications : first, the elastic modulus of the network scales with concentration like the osmotic pressure ; second, the hydrodynamic screening length scales like the excluded volume screening [8] length $\xi$ (eq. (2)).

Evidence for this dynamical correlation is determined from the time dependence of the observed autocorrelation function for the Rayleigh line in a light scattering experiment. The experimental set up is the same as described before in reference [1].

Since the network deformation corresponds to a well defined range of frequencies, it is important that the experiment be carried out in the proper momentum transfer $k=(4 \pi / \lambda) \sin (\theta / 2)$ domain. 
Following reference [7] the smallest momentum $k_{\min }$ is directly obtained form $T_{\mathrm{R}}$

$$
D_{\mathrm{c}} k_{\min }^{2} T_{\mathrm{R}}=1
$$

which gives

$$
k_{\min } R_{\mathrm{F}}=\left(C^{*} / \dot{C}\right)^{9 / 8}
$$

where $R_{\mathrm{F}}$ is the coil radius of giration at zero concentration; the concentration $C^{*}$ corresponds to the situation where the average distance between neighbour coils is equal to $R_{\mathrm{F}}$, i.e. the coils begin to overlap.

The upperlimit of the momentum range is given by

$$
k_{\max }=\xi^{-1}=R_{\mathrm{F}}^{-1}\left(C / C^{*}\right)^{3 / 4} .
$$

Beyond $k_{\max }$ the deformation modes are no longer cooperative; they propagate along each individual chain, without any contribution to the network. Such modes have been observed [2] in very dilute solutions, for which however there are no collective deformations.

The appropriate $k$ range is shown in figure 1 , in between $k_{\max }$ and $k_{\min }$, as a function of $C / C^{*}$. The $k R_{\mathrm{F}}$ versus $C / C^{*}$ plane is further divided into 4 regions : below $k_{\min }$ and $k R_{\mathrm{F}}=1$ (respectively II and $\mathrm{II}^{\prime}$ ), corresponding to diffusion of the center of mass ; above $k_{\max }$ and $k R_{\mathrm{F}}=1$ (respectively III

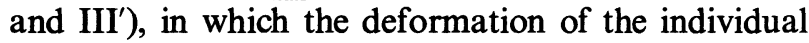
chains begin to contribute to the decay of the autocorrelation function.

Theory [7] thus predicts that in region I, figure 1 :

a) the autocorrelation function is a single exponential function of time ;

$b$ ) the inverse relaxation time $\tau^{-1}$ is proportional to $k^{2}$

$$
\tau^{-1}=D_{\mathrm{c}} k^{2},
$$

c) the diffusion coefficient is independant of molecular weight and varies with concentration as given by (1), (2), i.e. as $C^{3 / 4}$.

In region II, propositions $a$ ) and $b$ ) are known to

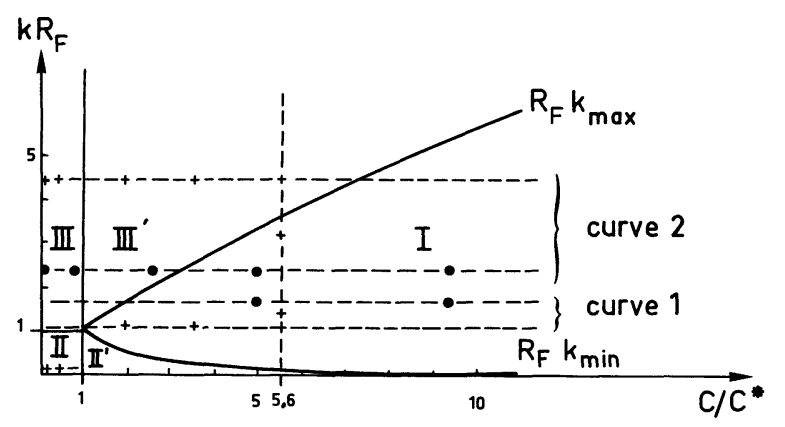

FIG. 1. - Representation of the range of concentration and momentum transfer for the predicted existence of cooperative diffusive modes (I). The other regions correspond to diffusive motion of the coil center of mass (II and $\mathrm{II}^{\prime}$ ), and to a superposition of center of mass motion and coil deformation (III and III'). The horizontal lines indicate the $k$ values of the experiment + sample 1 ; • sample 2 . hold true ; but the diffusion coefficient is essentially independent of concentration and decreases with molecular weight. By contrast, in region III, theory and experiment have shown [2] that the autocorrelation function must be fitted with at least two exponential functions.

The autocorrelation function was measured :

1) at constant $k R_{\mathrm{F}}$, for different values of $C / C^{*}$ (horizontal lines in figure 1);

2) at constant $C / C^{*}$, for different values of $k R_{\mathrm{F}}$ (vertical line in figure 1).

The polymer used in this experiment is polystyrene and the solvent is benzene. Characteristics of the system are given in table I.

\section{TABLE I}

Description of the polystyrene sample in benzene solution

$$
\begin{array}{cccc}
\text { Sample } & M_{\mathrm{W}} & C^{* \%} \% & R_{\mathrm{F}}(\mathrm{cm}) \\
- & - & - & - \\
1 & 3.8 \times 10^{6} & 4.25 \times 10^{-3} & 1.19 \times 10^{-6} \\
2 & 1.27 \times 10^{6} & 10^{-2} & 6.21 \times 10^{-6}
\end{array}
$$

In this concentration range of interest $\left(C \geqslant C^{*}\right)$ the solution contains many dust particles trapped by the network, whose effect can be seen on the light beam through the sample. The signal of the light scattering experiment is interpreted as heterodyne, the local oscillator being the light scattered elastically by these dust particles. In the range $C<C^{*}$, the low viscosity of the solution allows the dust particles to fall to the bottom of the cell, so the signal is interpreted as homodyne. In order to match the observed values, the relaxation times obtained in the dilute range are multiplied by a factor two.

In all cases, the autocorrelation function was fitted with a single exponential. The quality of the fit in region I is about the same as in region II. We have not tried to improve the fit in region III, since this is not the primary purpose of the experiment.

A typical result is shown in figure 2, where the inverse relaxation times are given as a function of $C$. The value of $\tau^{-1}$, measured at different $k$ values are normalized to the same nominal $k$ value $\left(k_{\mathrm{N}}\right)$ according to the rule

$$
\tau^{-1}=\tau_{\exp }^{-1} k_{\mathrm{N}}^{2} / k^{2}
$$

where $\tau_{\exp }^{-1}$ is the value measured at $k$ and where

$$
k_{\mathrm{N}}=3.86 \times 10^{5} \mathrm{~cm}^{-1} \text {. }
$$

Curve 1 in figure 2 corresponds to the lower values of $k R_{\mathrm{F}}$ (see Table II). Below $C^{*}$, the inverse relaxation time $\tau^{-1}$ is essentially constant : it is identified as the diffusion coefficient of the individual coils. Beyond $C^{*}$, the values of $\tau^{-1}$ are seen to increase : the diffusion is cooperative. Thus as predicted by 


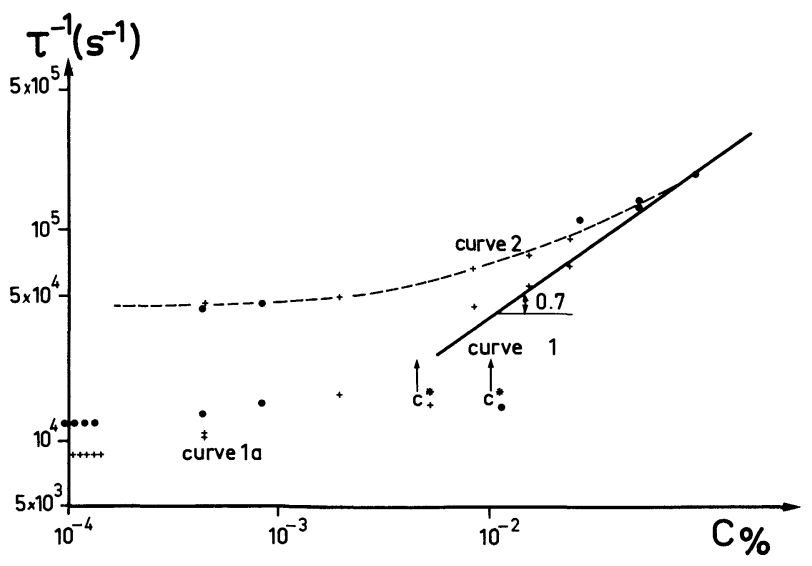

Fig. 2. - Normalized inverse relaxation times as a function of concentration : $\tau^{-1}=\tau_{\exp }^{-1} k_{\mathrm{N}}^{2} / k^{2}$ where $\tau_{\exp }, k^{2}$ are measured values and $k_{\mathrm{N}}=3.86 \times 10^{5} \mathrm{~cm}^{-1}$, + sample 1 , sample 2 . Curve 1 : measured at $k R_{\mathrm{F}} \sim 1$. Curve 2 : measured at $k R_{\mathrm{F}}>1$; +++++ normalized inverse relaxation time exptrapolated at $C=0$, sample 1 . $\bullet \bullet \bullet$ normalized inverse relaxation time extrapolated at $C=0$, sample 2 .

TABLE II

Description of the experiments

\begin{tabular}{|c|c|c|c|c|}
\hline Sample & $C \%$ & $k R_{\mathrm{F}}$ & $\tau_{\exp }^{-1}\left(s^{-1}\right)$ & $\tau_{\text {normalized }}^{-1}\left(s^{-1}\right)$ \\
\hline 一 & - & - & - & - \\
\hline \multirow{11}{*}{1} & \multirow{2}{*}{$2.38 \times 10^{-2}$} & 4.5 & $9.11 \times 10^{4}$ & $9.11 \times 10^{4}$ \\
\hline & & 1.1 & $4.11 \times 10^{3}$ & $6.78 \times 10^{4}$ \\
\hline & \multirow{2}{*}{$1.53 \times 10^{-2}$} & 4.5 & $7.62 \times 10^{4}$ & $7.62 \times 10^{4}$ \\
\hline & & 1.1 & $3.24 \times 10^{3}$ & $5.35 \times 10^{4}$ \\
\hline & \multirow{2}{*}{$8.32 \times 10^{-3}$} & 4.5 & $6.66 \times 10^{4}$ & $6.66 \times 10^{4}$ \\
\hline & & 1.1 & $2.73 \times 10^{3}$ & $4.36 \times 10^{4}$ \\
\hline & \multirow{3}{*}{$1.92 \times 10^{-3}$} & 4.5 & $4.79 \times 10^{4}$ & \\
\hline & & 0.2 & $3.11 \times 10^{4}$ & $1.63 \times 10^{4}$ \\
\hline & & 4.5 & $4.48 \times 10^{4}$ & $4.48 \times 10^{4}$ \\
\hline & \multirow[t]{2}{*}{$4.44 \times 10^{-4}$} & 1.1 & $6.85 \times 10^{2}$ & $1.09 \times 10^{4}$ \\
\hline & & 0.2 & $1.97 \times 10^{1}$ & $1.06 \times 10^{4}$ \\
\hline \multirow{11}{*}{2} & $\dot{C} \rightarrow 0$ & & & $8.55 \times 10^{3}\left(^{*}\right)$ \\
\hline & \multirow{2}{*}{$9.37 \times 10^{-2}$} & 2.4 & $1.86 \times 10^{5}$ & $1.86 \times 10^{5}$ \\
\hline & & 1.7 & $9.24 \times 10^{4}$ & $1.83 \times 10^{5}$ \\
\hline & \multirow{2}{*}{$5.02 \times 10^{-2}$} & 2.4 & $1.28 \times 10^{5}$ & $1.28 \times 10^{5}$ \\
\hline & & 1.7 & $6.91 \times 10^{4}$ & $1.39 \times 10^{5}$ \\
\hline & $2.66 \times 10^{-2}$ & 2.4 & $1.11 \times 10^{5}$ & $1.11 \times 10^{5}$ \\
\hline & \multirow{2}{*}{$8.37 \times 10^{-4}$} & 2.4 & $4.49 \times 10^{4}$ & $148 \times 10^{4}$ \\
\hline & & 0.2 & $1.04 \times 10^{2}$ & \\
\hline & \multirow{2}{*}{$4.37 \times 10^{-4}$} & 2.4 & $4.25 \times 10^{4}$ & $136 \times 10^{4}$ \\
\hline & & 0.2 & $9.51 \times 10^{1}$ & $1.00 \times 10$ \\
\hline & $\mathrm{C} \rightarrow 0$ & & & $1.21 \times 10^{4}\left({ }^{*}\right)$ \\
\hline
\end{tabular}

(*) The values of $\tau^{-1}$ normalized in the limit $\mathbf{C} \rightarrow 0$ are obtained from the diffusion coefficients reported in reference [1].

de Gennes, in semi-dilute solutions, the observed diffusion coefficient increases with concentration. We also notice that two different molecular weights yield the same $\tau^{-1}$ values in region $\mathrm{I}$. This is to be compared to the molecular weight dependence in region II (Fig. 2, curve 1a). The slope of curve 1 in region $I$ is $0.7 \pm 0.05$, to be compared to the theoretical value, 0.75 .

Curve 2 corresponds to values of $k R_{\mathrm{F}}$ far above unity. This a border line case, since only a few points of this curve belong to region I. The effect of coil deformation modes at low concentration is clearly seen in region III : the $\tau^{-1}$ values have increased. The exact contribution of center of mass diffusion and individual coil deformation cannot be determined from these data, since we have interpreted the autocorrelation function with a single exponential (for $k R_{\mathrm{F}}$ greater than unity, this procedure is meanningful only inside region $\mathrm{I})$. As in case of curve $1, \tau^{-1}$ increases with $C$ beyond $C^{*}$. The slope is however much smaller $(0.42)$. This fact may be attributed to the contribution of the individual coil deformation modes, as if they coexisted with the cooperative modes. There would be a strong cross over effect in reciprocal space. This point is illustrated by figure 3 , where data at constant $C / C^{*}$ are plotted as a function of $k$. One would expect $\tau^{-1} / k^{2}$ to be independent of $k$ in region I. In fact this ratio increases slightly as $k$ increases. The difficulty in interpreting

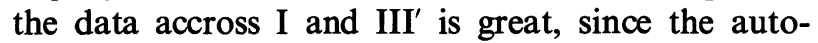
correlation functions have very different analytical behaviours in I and III' respectively (ref. [7]).

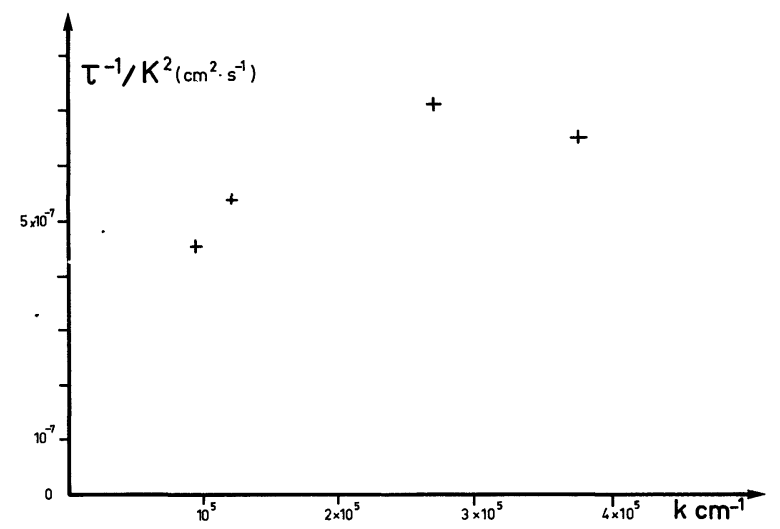

FIG. 3. - Inverse relaxation times divided by $k^{2}$, for different $k$ values, at $C / C^{*}=5.6$.

Starting from the boundary $C / C^{*}=1$, we have tested the network properties of the semi-dilute polymer solutions by light scattering experiments.

The cooperative diffusion coefficient which is directly obtained from the decay of the autocorrelation increases with concentration.

This coefficient is identically the same for two molecular weights of the coils.

The situation is very different from the dilutesolution, for which the observed diffusion coefficient cleary decreases with molecular weight of the coil and for which there is no striking concentration dependence.

There is a difficulty in comparing experimental results and theoretical predictions, arising from the fact that the coil network is associated with a narrow 
range of frequencies in the vicinity of $C^{*}$. However the qualitative features observed clearly indicate that the coils form a network as soon as they overlap.

Note added in proof. - Dr. S. Candau has drawn our attention to the work of Rehage, G., Ernst, O. and Fuhrmann, J., discuss. Faraday Soc. 49 (1970) 208 in which the polymer concentration diffusion is reported at high concentrations and which indicates a behaviour related to the results pointed in this letter.

Acknowledgments. - We wish to thank Pr. P. G. de Gennes for suggesting this experiment and Dr. S. Candau for helpfull criticism of the manuscript.

\section{References}

[1] Adam, M., Delsanti, M., to be submitted to $J$. Physique.

[2] King, T. A., Knox, A., McAdam, J. D. G., Chem. Phys. Lett. 19 (1973) 351

[3] Munch, J. P., Candau, S., Duplessix, R., Picot, C., Benoit, H., J. Physique Lett. 35 (1974) 239.

See also DupPLESSIX, R., Thesis. Strasbourg (1975).

[4] FerRY, J. D., Viscoelastic properties of polymers, New York, London (J. Wiley) 1961.

[5] Janeschitz-Kriegel, H., Adv. Polymer Sci. 6 (1969) 170.

[6] Peterlin, A., Pure Appl. Chem. 12 (1966) 563.

[7] De Gennes, P. G., to be submitted to Macromolecules.

[8] Daoud, M., Cotton, J. P., Farnoux, B., Jannink, G., Sarma, G., Benoit, H., Duplessix, C., Picot, C. et De GenNes, P. G., Macromolecules 8 (1975) 804. 